\title{
THE OPERATION OF THE UNIVERSAL DOMAIN ON THE PLANE ${ }^{1}$
}

\author{
SHERWOOD EBEY
}

The problem we are considering is if

$$
(t, x, y) \rightarrow(P(t, x, y), Q(t, x, y))
$$

defines a regular operation of the additive group of the universal domain $\Omega$ on the affine plane $A^{2}$, then what can be said about the form of the polynomials $P$ and $Q$ ?

First we note that any change of coordinates for $A^{2}$ is defined by equations

$$
\begin{array}{ll}
u=M(x, y), & x=R(u, v), \\
v=N(x, y), & y=S(u, v),
\end{array}
$$

where $M, N, R, S$ are polynomials with coefficients in $\Omega$. Now the operation of $\Omega$ on $A^{2}$ given by equations (1) is defined in $u v$-coordinates by

(3) $\quad(t, u, v) \rightarrow(M(P(t, R, S), Q(t, R, S)), N(P(t, R, S), Q(t, R, S)))$.

We shall use a theorem from a paper by Engel [1] which states that in a change of coordinates such as (2) the degree of $M$ must divide the degree of $N$ or vice versa. Engel's proof is for characteristic $=0$. Assuming this result we will prove the following theorem.

THEOREM. If the characteristic of $\Omega$ is 0 and if there is a regular operation of $\Omega$ on $A^{2}$, then there is a change of coordinates of $A^{2}$ such that in terms of the uv-coordinates the given operation has the form

$$
(t, u, v) \rightarrow(u, v+t f(u)) \quad \text { with } f \in \Omega[u] .
$$

We will use the theory of algebraic groups as developed by Rosenlicht in [2]. If $H \in \Omega(x, y)$ and $t \in \Omega$, then $\lambda_{t} H$ is the function $H(P(t, x, y), Q(t, x, y)) . H$ is called invariant if $\lambda_{t} H=H$ for all $t \in \Omega$. Now to prove the theorem we proceed by proving a sequence of three lemmas.

Lemma 1. There exists a nonconstant polynomial in $\Omega[x, y]$ which is invariant.

PROof. Let $k$ be an algebraically closed field of definition for $\Omega, A^{2}$,

Received by the editors September 1, 1961.

1 This paper is based on a portion of the author's doctoral dissertation, written at Northwestern University under the supervision of Professor M. Rosenlicht. 
and the operation on $A^{2}$. Consider the variety $W$ of $\Omega$-orbits on $A^{2}$. If this were of dimension 0 , any two independent generic points for $A^{2}$ over $k$ would belong to the same orbit. This is impossible and therefore $\operatorname{dim}(W)>0$.

Now $\operatorname{dim}(W)>0$ implies that the field $k(W)$ has a nonconstant function. But $k(W)$ is $k$-isomorphic to the subfield of invariant functions in $k(x, y)$. Hence there exists a nonconstant function $H$ in $k(x, y)$ which is invariant.

Let $H=H_{1} / H_{2}$ where $H_{i} \in k[x, y]$ and $H_{1}$ and $H_{2}$ are relatively prime. Let $t$ be a variable over $k(x, y)$. Now $\lambda_{t} H=H$ implies $\lambda_{t} H_{1} / \lambda_{t} H_{2}$ $=H_{1} / H_{2}$. Using the unique factorization of $k[t, x, y]$ it can be shown that $\lambda_{t} H_{i}=H_{i}$. Since $H$ is a nonconstant function, either $H_{1}$ or $H_{2}$ is the required nonconstant invariant polynomial in $k[x, y]$. Q.e.d.

Note that by taking powers of such an invariant polynomial we can obtain an invariant polynomial of arbitrarily high degree.

For the operation defined by polynomials (1) we must have $P(0, x, y)=x$ and $Q(0, x, y)=y$. Therefore these polynomials must be of the form

$$
\begin{aligned}
& P(t, x, y)=x+t f_{1}(x, y)+t^{2} f_{2}(x, y)+\cdots+t^{n} f_{n}(x, y), \\
& Q(t, x, y)=y+t g_{1}(x, y)+t^{2} g_{2}(x, y)+\cdots+t^{m} g_{m}(x, y) .
\end{aligned}
$$

Lemma 2. If the polynomials $P$ and $Q$ which define the operation of $\Omega$ on $A^{2}$ are such that $f_{n} \neq 0, g_{m} \neq 0,0<n \leqq m$, and $m / n=l / j$ with $(l, j)=1$, then $f_{n}^{l}=c g_{m}^{j}$ where $c$ is a constant.

Proof. Let the weight of a monomial $x^{\nu} y^{\mu}$ be defined as the integer $n \nu+m \mu$. The weight of a polynomial will be the maximum of the weights of its monomials. Now

$$
\lambda_{t}\left(x^{\nu} y^{\mu}\right)=P^{\nu} Q^{\mu}=\left(f_{n}^{\nu} g_{m}^{\mu}\right) t^{n \nu+m \mu}+\text { terms of lower degree in } t .
$$

By Lemma 1 there is a nonconstant polynomial $H(x, y)$ such that $\lambda_{t} H=H$. If $s$ is the weight of $H$ and if $x^{v} y^{\mu}$ is the monomial of $H$ of weight $s$ and with degree in $x$ maximal, then the part of $H$ of weight $s$ must be of the form

$$
H_{s}(x, y)=a_{0} x^{\nu} y^{\mu}+a_{1} x^{\nu-l} y^{\mu+j}+a_{2} x^{\nu-2 l} y^{\mu+2 j}+\cdots+a_{q} x^{\nu-q l} y^{\mu+q j} .
$$

The coefficient of $t^{s}$ in $\lambda_{t} H$ will be $H_{s}\left(f_{n}, g_{m}\right)$ which must be identically zero since $\lambda_{t} H=H$. From this we obtain a relation of the form

$$
a_{0} f_{n}^{q l}+a_{1} f_{n}^{(q-1) l} g_{m}^{j}+\cdots+a_{q} g_{m}^{q j}=0 .
$$

This implies that $f_{n}^{l} / g_{m}^{j}$ must equal one of the roots of an algebraic 
equation of degree $q$. Therefore $f_{n}^{l}=c g_{m}^{s}$ where $c$ is some constant. Q.e.d.

Lemma 3. If the polynomials $P$ and $Q$ in (4) are such that $f_{n} \neq 0$, $g_{m} \neq 0,0<n \leqq m$, then $n \mid m$.

Proof. Choose an invariant polynomial $H(x, y)$ so that the degree of $H$ is greater than the maximum of the degrees of

$$
\left\{f_{1}, f_{2}, \cdots, f_{n}, g_{1}, g_{2}, \cdots, g_{m}\right\} .
$$

Let $\nu$ be the degree of $H$.

Lemma 2 implies that if $f_{n}$ and $g_{m}$ are nonconstant polynomials, then $g_{m}=G^{l}(x, y)$ and $f_{n}=c G^{j}(x, y)$ where $c$ is a constant and $l$ and $j$ are as in Lemma 2. Let $\mu$ be the degree of $G$. (If $f_{n}$ and $g_{m}$ are constants, $\mu=0$.)

Now since $(t, x, y) \rightarrow(P(t, x, y), Q(t, x, y))$ defines a regular operation of $\Omega$ on $A^{2}$, it follows that

$$
\Omega[t, x, y]=\Omega[t, P(t, x, y), Q(t, x, y)] .
$$

If we let $t=H(x, y)$ in (5) we have that

$$
\Omega[x, y]=\Omega[H, x, y]=\Omega[H, P(H, x, y), Q(H, x, y)] .
$$

Since $H$ is invariant $H(x, y)=H(P(H, x, y), Q(H, x, y))$. Thus (6) implies

$$
\Omega[x, y]=\Omega[P(H, x, y), Q(H, x, y)] .
$$

From the choice of $\nu$ it follows that the degree of the first generator on the right side of (7) must be the degree of $H^{n} f_{n}$ which is $n \nu+j \mu$. Similarly the degree of the second generator on the right side of (7) is the degree of $H^{m} g_{m}$ which is $m \nu+l_{\mu}$.

By the result in [1] that we referred to above, $(n \nu+j \mu) \mid(m \nu+l \mu)$. This implies that $j \mid l$. But since $(j, l)=1, j=1$ and $n \mid m$. Q.e.d.

We will now prove the theorem. Suppose we have polynomials $P$ and $Q$ of form (4) with $n \neq 0$ and $m \neq 0$. Let $n \leqq m$. By Lemma 3 we know that $n \mid m$ so that in the notation of Lemma $2, m / n=l$ and $j=1$. Thus Lemma 2 implies that $g_{m}=c f_{n}^{l}$ where $c$ is a constant.

Make the following change of coordinates for $A^{2}$ :

$$
\begin{array}{ll}
u=x, & x=u, \\
v=y-c x^{l}, & y=v+c u^{l} .
\end{array}
$$

Applying equation (3) to this particular change of coordinates, the 
given operation will be defined in $u v$-coordinates by

(8) $(t, u, v) \rightarrow\left(P\left(t, u, v+c u^{l}\right), Q\left(t, u, v+c u^{l}\right)-c P^{l}\left(t, u, v+c u^{l}\right)\right)$.

The coefficient of $t^{m}$ in $Q\left(t, u, v+c u^{l}\right)-c P^{l}\left(t, u, v+c u^{l}\right)$ is $g_{m}\left(u, v+c u^{l}\right)$ $-c f_{n}^{l}\left(u, v+c u^{l}\right)$, which is zero.

Hence (8) reduces to an expression of form (4) in which the degree of $P$ in $t$ is $n$ while the degree of $Q$ in $t$ is less than $m$. We can repeat this process until we obtain coordinates in which either $P$ or $Q$ will have degree 0 in $t$.

Thus for any regular operation of $\Omega$ on $A^{2}$, there are coordinates $u, v$ so that the operation has the form

$$
(t, u, v) \rightarrow(u, Q(t, u, v))
$$

But we may consider $(t, v) \rightarrow(Q(t, u, v))$ as defining an operation on the line. Now $Q$ must satisfy the identity

$$
Q(s+t, u, v)=Q(s, u, Q(t, u, v)) \text {. }
$$

Since characteristic $=0$, it follows that $Q(t, u, v)$ must be of the form $v+t f(u)$ where $f \in \Omega[u]$. Thus in $u v$-coordinates the given operation has the form

$$
(t, u, v) \rightarrow(u, v+t f(u)) .
$$

This completes the proof of the theorem.

\section{REFERENCES}

1. W. Engel, Ganze Cremona-Transformationen von Primzahlgrad in der Ebene, Math. Ann 136 (1958), 319-325.

2. M. Rosenlicht, Some basic theorems on algebraic groups, Amer. J. Math. 78 (1956), 401-443.

Wheaton College, Wheaton, Illinois 